\title{
Design of an OFDM Cooperative Space-Time Diversity System
}

\author{
Oh-Soon Shin, Albert M. Chan, Member, IEEE, H. T. Kung, and Vahid Tarokh, Senior Member, IEEE
}

\begin{abstract}
In this paper, we propose a wireless system that realizes theoretical benefits of space-time cooperation. Specifically, we design a space-time cooperative system based on orthogonal frequency division multiplexing (OFDM), which we refer to as a cooperative (CO)-OFDM system. Our design includes a two-phase space-time cooperation protocol, as well as a transmitter and receiver architecture that facilitates cooperation. Furthermore, we devise a frame structure, on which we build practical timing and frequency synchronization algorithms and a channel estimation algorithm. In particular, the proposed frequency synchronization algorithm utilizes the underlying structure of the cooperation protocol, and the proposed channel estimation algorithm is based on a pairwise orthogonal construction of two sequences. We validate the performance of the proposed synchronization and channel estimation algorithms through simulations. We then present simulation results that demonstrate the overall performance advantage of the CO-OFDM system over an OFDM system without cooperation, not only under idealistic assumptions but also under realistic situations where the proposed algorithms are employed.
\end{abstract}

Index Terms-Channel estimation, cooperative diversity, frequency offset, orthogonal frequency division multiplexing (OFDM), synchronization.

\section{INTRODUCTION}

$\mathbf{S}$ PACE-TIME coding realizes spatial diversity by transmitting signals through multiple transmit antennas [1], [2]. However, the need for multiple transmit antennas is a prohibiting factor in deploying such technique in certain wireless devices, such as portable terminals and wireless sensors, due to size, cost, and computational power limitations. In this case, a promising approach for achieving spatial diversity without multiple antennas is space-time cooperation, which is also known as cooperative diversity [3]-[9]. The fundamental principle is to effectively establish multiple antennas in a distributed manner by allowing neighboring nodes to share their radio resources. Space-time cooperation can be applied to a broad range of

Manuscript received January 15, 2006; revised May 29, 2006 and June 4, 2006. This work was supported in part by the Air Force Office of Scientific Research under Contract FA9550-04-C-0076. The work of O.-S. Shin was also supported in part by the Ministry of Information and Communication, Korea. The review of this paper was coordinated by Dr. A. Ghrayeb.

O.-S. Shin was with the Division of Engineering and Applied Sciences, Harvard University, Cambridge, MA 02138 USA. He is now with the Samsung Electronics Company, Ltd., Suwon 443-742, Korea (e-mail: ohsoon.shin@ samsung.com).

A. M. Chan is with Vanu, Inc., Cambridge, MA 02142 USA (e-mail: chanal@vanu.com).

H. T. Kung and V. Tarokh are with the Division of Engineering and Applied Sciences, Harvard University, Cambridge, MA 02138 USA (e-mail: kung@harvard.edu; vahid@deas.harvard.edu).

Color versions of one or more of the figures in this paper are available online at http://ieeexplore.ieee.org.

Digital Object Identifier 10.1109/TVT.2007.897642 applications, such as broadcast networks, sensor networks, and relay networks. In particular, in a relay network, traditional relays simply forward signals after amplifying or decoding [10], [11]. In the context of space-time cooperation, however, relays are allowed to cooperate with one another and the original source to form more reliable transmit signals.

A variety of cooperative diversity protocols have been studied and analyzed to demonstrate the potential benefits of spacetime cooperation [3]-[9]. These works have shown that much of the diversity gain of the original space-time coding can be achieved through well-designed protocols. However, most of these works have assumed perfect timing and frequency synchronization. As discussed in [12] and [13], handling of timing and frequency offsets is one of the most critical and challenging issues in implementing a space-time cooperative system. Timing offsets are primarily caused by the geometrically separated nature of the transmit nodes, which leads to different propagation delays for different wireless links. On the other hand, frequency offsets result when different nodes are driven by oscillators with nonidentical characteristics.

Space-time cooperation should be implemented on top of a transmission technology, such as direct-sequence spreadspectrum (SS) and orthogonal frequency division multiplexing (OFDM). In particular, OFDM is one of the most promising technologies for current and future wireless communications. Various forms of OFDM have been adopted in wireless standards, for example, digital audio/video broadcasting [14], IEEE 802.11a [15], and IEEE 802.16 [16]. In OFDM transmission, the total bandwidth is divided into a number of subcarriers, over which multiple data symbols are transmitted in parallel. Therefore, the symbol duration of an OFDM signal increases with the number of subcarriers. From the viewpoint of spacetime cooperation, the longer symbol duration along with the use of cyclic prefix makes the system robust to timing errors. However, at the same time, longer symbol duration causes OFDM signals to become more sensitive to frequency offsets. Moreover, frequency synchronization techniques developed for conventional OFDM systems, such as the schemes in [17]-[19], may not be applicable to cooperative systems. Therefore, development of a frequency synchronization algorithm is essential when designing OFDM-based cooperative systems. On the other hand, it is noteworthy that the use of cooperation for OFDM systems will be particularly useful in channels with scarce frequency selectivity, which are encountered in many indoor environments.

In this paper, we design a space-time cooperative system based on OFDM, which we refer to as a cooperative (CO)OFDM system. Our design includes most of the physical layer 

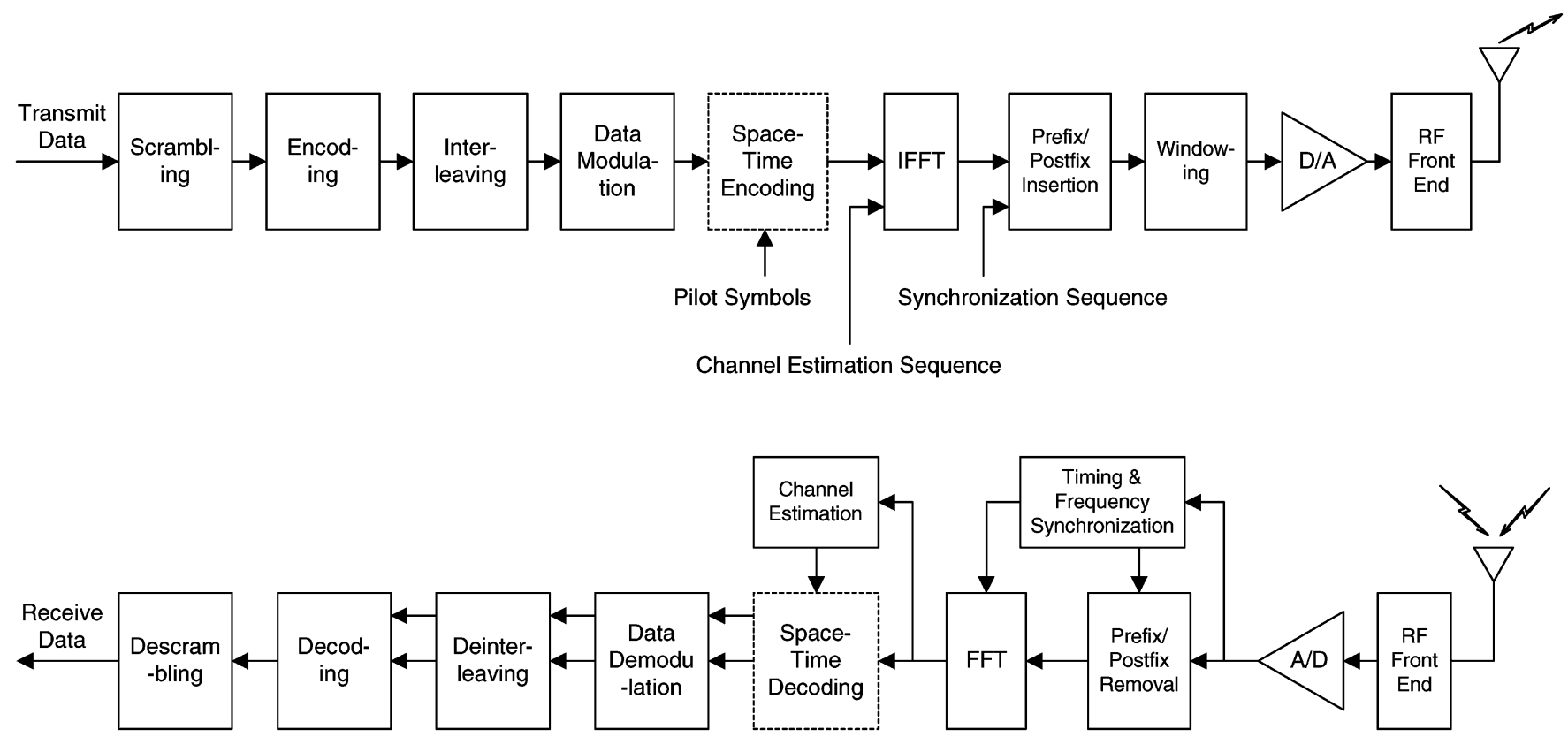

Fig. 1. Block diagram of the CO-OFDM transmitter and receiver (dotted blocks are used only in the cooperation phase).

components, including the transmitter and receiver architecture, the frame structure, and the space-time cooperation protocol. The cooperation protocol is based on a two-phase transmission of each frame, as considered in [8] and [9]. Based on the frame structure, we develop practical timing and frequency synchronization algorithms, and a channel estimation algorithm for CO-OFDM systems. In particular, the proposed frequency synchronization algorithm makes use of the underlying twophase cooperation protocol, and the proposed channel estimation algorithm is based on a pairwise orthogonal construction of two sequences. The performance of the CO-OFDM system is extensively evaluated through simulations.

This paper is organized as follows. Section II describes the proposed CO-OFDM system. We develop timing and frequency synchronization algorithms in Section III and a channel estimation algorithm in Section IV. In Section V, we provide simulation results that demonstrate the performance benefits of the CO-OFDM system. Finally, conclusions are drawn in Section VI.

\section{CO-OFDM SYSTEM}

We consider a communication scenario where only one relay node helps the source node deliver information data to the destination node. Each node is assumed to transmit or receive signals with only one antenna at the same frequency.

\section{A. Transmitter and Receiver}

Fig. 1 illustrates a block diagram of the CO-OFDM transmitter and receiver. The structure of the transmitter is similar to that of the IEEE 802.11a standard [15]. Data bits are first scrambled by a side-stream scrambler with four different seed values as in [15], and they are encoded using a nonrecursive convolutional encoder. Encoded bits are interleaved by an $n_{\text {row }} \times n_{\text {col }}$ block interleaver and then modulated into transmit symbols by a given modulation scheme. When transmitting cooperation subframes, which will be explained in Section II-B, transmit symbols are encoded according to a space-time cooperation protocol. A series of $N_{d}$ symbols are loaded on OFDM data subcarriers, and $N_{p}$ pilot symbols and $N_{g}$ guard or null symbols are loaded on corresponding subcarriers. Thus, the total number of subcarriers is $N=N_{d}+N_{p}+N_{g}$, among which $N_{u}=$ $N_{d}+N_{p}$ subcarriers are dedicated to data or pilots. An inverse fast Fourier transform (IFFT) of the $N$ symbols results in $N$ samples of time domain signals. A cyclic prefix of $L_{\mathrm{cp}}$ samples is appended in front of the $N$ time domain samples to form a complete OFDM symbol. To implement transmit windowing, additional cyclic prefix and postfix samples are then attached [14]. Each OFDM symbol is multiplied by a window function, and a few boundary samples of the OFDM symbol are overlapped and added with those of adjacent OFDM symbols. The windowed samples are finally converted from digital to analog with the sample duration $t_{\mathrm{s}}=1 / W$ before going through the RF chain, where $W$ is the total transmission bandwidth. For simplicity of signal descriptions, we ignore transmit windowing in this paper, since it does not affect the error performance when the timing error and delay spread are less than the length of cyclic prefix. Correspondingly, each OFDM symbol consists of $L_{\text {sym }}=N+L_{\text {cp }}$ samples. The receiver first establishes timing and frequency synchronization and then estimates channel responses using the received preamble signals. Then, each OFDM symbol is demodulated according to the inverse procedure of the transmitter. In particular, the soft-decision Viterbi algorithm [20] is employed to decode the convolutionally encoded data bits.

\section{B. Frame Structure}

In order to realize space-time cooperation, each frame of the CO-OFDM system is divided into two subframes, as illustrated in Fig. 2. We refer to these two subframes as the listening subframe and the cooperation subframe. Each subframe 


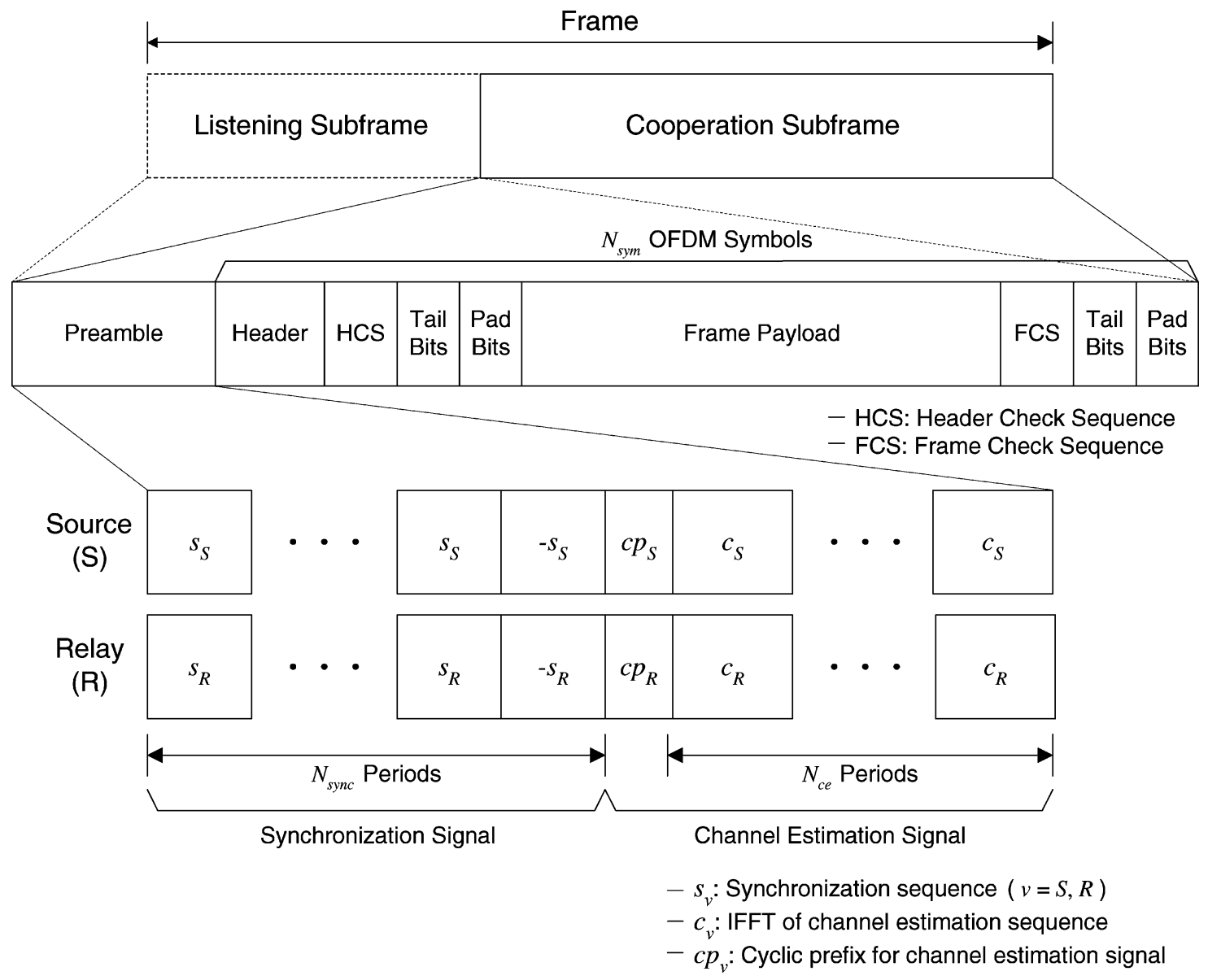

Fig. 2. Frame structure of the CO-OFDM system.

comprises of the same signal components: preamble, header, header check sequence (HCS), data payload, frame check sequence (FCS), tail bits, and pad bits. Specifically, the preamble is split into two parts, as depicted in Fig. 2. Note that the two subframes can have different length. Synchronization signals are composed of $N_{\mathrm{sync}}-1$ periods of a synchronization sequence $\left\{s_{v}[n]: 0 \leq n<L_{\text {sync }}\right\}$ and one period of $\left\{-s_{v}[n]\right\}$, where the subscript $v$ stands for a transmit node that uses the sequence, i.e., $v=S$ and $v=R$ correspond to the source and relay, respectively. The sequences $\left\{s_{S}[n]\right\}$ and $\left\{s_{R}[n]\right\}$ are desired to have both good autocorrelation and cross correlation properties for easy synchronization. Channel estimation signals are constructed by repeating a sequence $\left\{c_{v}[n]: 0 \leq n<\right.$ $N\} N_{\text {ce }}$ times and attaching a cyclic prefix of $N_{\text {ce }} L_{\text {cp }}$ samples, as in [15]. The sequence $\left\{c_{v}[n]\right\}$ is obtained by an IFFT of the channel estimation sequence $\left\{C_{v}[k]: 1 \leq|k| \leq N_{u} / 2\right\}$. Note that the destination needs to estimate channel responses of two distinct links when receiving cooperation subframes. To make these estimations feasible, we enforce $\left\{C_{S}[k]\right\}$ and $\left\{C_{R}[k]\right\}$ to be orthogonal over every pair of adjacent subcarriers. To be specific, elements in each partition comprised of two successive elements of $\left\{C_{S}[k]\right\}$ have the same magnitude: $\left|C_{S}[k]\right|=\left|C_{S}[k+1]\right|$ for odd $k$, and $\left\{C_{R}[k]\right\}$ is formed by flipping the sign of $\left\{C_{S}[k]\right\}$ only at even subcarrier indexes: $C_{R}[k]=-C_{S}[k]$, if $k$ is even, and $C_{R}[k]=C_{S}[k]$, otherwise. If Golay sequences are to be employed as channel estimation sequence due to their favorable peak to mean envelope power ratio, then it is noteworthy that if $\left\{C_{S}[k]\right\}$ is a Golay sequence, then so is $\left\{C_{R}[k]\right\}$ [21]. The header contains information on the following frame payload, such as transmission rate, payload length, and scrambler seed. HCS and FCS are generated using the 16-bit CRC-CCITT [22]. The tail bits are zero bits that are needed to return the convolutional encoder into zero state [20]. The pad bits are also zero bits that are inserted to align the data stream on OFDM symbol boundaries. The data bits from the header to the end of a subframe form $N_{\text {sym }}$ OFDM symbols, of which the first two symbols are attributed to the header part.

\section{Signal and Channel Models}

We first establish some notation. Let the subscript $v=$ $S, R, D$ stand for the source, relay, and destination nodes, respectively, and let subscripts $\ell=S D, S R, R D$ stand for the $S D$ link, $S R$ link, and $R D$ link, as illustrated in Fig. 3. Let $\mathbb{I}_{d}$, $\mathbb{I}_{p}, \mathbb{I}_{g} \subset\{-N / 2,-N / 2+1, \ldots, N / 2-1\}$ denote the sets of subcarrier indexes corresponding to data subcarriers, pilot subcarriers, and guard or null subcarriers, respectively, and let $\mathbb{I}_{u}=\mathbb{I}_{d} \cup \mathbb{I}_{p}$. The cardinalities of these index sets are $\left|\mathbb{I}_{d}\right|=$ $N_{d},\left|\mathbb{I}_{p}\right|=N_{p},\left|\mathbb{I}_{g}\right|=N_{g}$, and $\left|\mathbb{I}_{u}\right|=N_{u}$. For notational convenience, we assume that neither the FFT nor IFFT operation changes the signal power and that the preamble sequences and the data symbols are normalized to have unit power. We use small letters to represent time domain signals, and capital letters to represent frequency domain signals. 


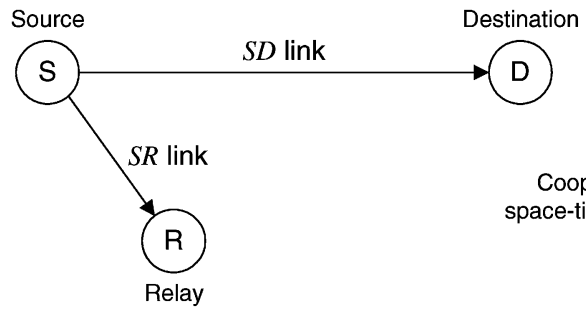

Listening Phase

\section{Listening Phase}

IEEE TRANSACTIONS ON VEHICULAR TECHNOLOGY, VOL. 56, NO. 4, JULY 2007

Fig. 3. Link establishments in each phase of the space-time cooperation protocol.

According to the frame structure in Section II-B, for node $v=S$ or $R$, the lowpass equivalent of the transmit signal $u_{v}(t)$ for each subframe can be expressed as

$$
\begin{aligned}
u_{v}(t)= & \sqrt{P_{v}} \sum_{n=0}^{L_{\mathrm{sync}}\left(N_{\mathrm{sync}}-1\right)-1} s_{v}[n] p\left(t-n t_{\mathrm{s}}\right) \\
& -\sqrt{P_{v}} \sum_{n=0}^{L_{\mathrm{sync}}-1} s_{v}[n] \\
& \times p\left(t-\left(n+L_{\mathrm{sync}}\left(N_{\mathrm{sync}}-1\right)\right) t_{\mathrm{s}}\right) \\
& +\sqrt{P_{v}} \sum_{n=0}^{L_{\mathrm{sym}} N_{\mathrm{ce}}-1} c_{v}\left[\left(n-L_{\mathrm{cp}} N_{\mathrm{ce}}\right) \bmod N\right] \\
& \times p\left(t-\left(n+L_{\mathrm{sync}} N_{\mathrm{sync}}\right) t_{\mathrm{s}}\right) \\
& +\sqrt{P_{v}} \sum_{m=0}^{N_{\mathrm{sym}}-1} \sum_{n=0}^{L_{\mathrm{sym}}-1} x_{v, m}\left[\left(n-L_{\mathrm{cp}}\right) \bmod N\right] \\
& \times p\left(t-\left(n+m L_{\mathrm{sym}}+L_{p}\right) t_{\mathrm{s}}\right)
\end{aligned}
$$

where $P_{v}$ denotes the transmit power of the node $v, L_{p} \triangleq$ $L_{\text {sync }} N_{\text {sync }}+L_{\text {sym }} N_{\text {ce }}$ denotes the preamble length in samples, and $p(t)$ is a rectangular pulse with duration $t_{\mathrm{s}}: p(t)=1$ if $0 \leq t<t_{\mathrm{s}}$, and 0 otherwise. The $L_{\text {sym }}$ samples constituting the $m$ th OFDM symbol $\left\{x_{v, m}[n]: 0 \leq n<L_{\text {sym }}\right\}$ are generated by an IFFT of the frequency domain symbols $\left\{X_{v, m}[k]: k \in\right.$ $\left.\mathbb{I}_{u} \cup \mathbb{I}_{g}\right\}$ and cyclic extension of $L_{\mathrm{cp}}$ samples.

The channel of each wireless link is assumed to be constant for the duration of each frame and allowed to vary between frames. The channel impulse response of each link is modeled as a tapped delay line with tap spacing equal to the sample duration $t_{\mathrm{s}}$ :

$$
h_{\ell}(\tau)=\sum_{q=0}^{Q_{\ell}-1} h_{\ell}[q] \delta\left(\tau-q t_{\mathrm{s}}\right), \quad \ell=S D, S R, R D
$$

where $Q_{\ell}$ denotes the number of resolvable paths for the link $\ell$, and $\delta(\cdot)$ denotes the Dirac delta function. The channel gain $h_{\ell}[q]$ of each path is assumed to follow an independent complex Gaussian distribution with zero mean. Geometric gains of the
$S R$ link and $R D$ link relative to the primary $S D$ link are defined as

$$
\begin{aligned}
& G_{S R} \triangleq \frac{E\left[\sum_{q=0}^{Q_{S R}-1}\left|h_{S R}[q]\right|^{2}\right]}{E\left[\sum_{q=0}^{Q_{S D}-1}\left|h_{S D}[q]\right|^{2}\right]} \\
& G_{R D} \triangleq \frac{E\left[\sum_{q=0}^{Q_{R D}-1}\left|h_{R D}[q]\right|^{2}\right]}{E\left[\sum_{q=0}^{Q_{S D}-1}\left|h_{S D}[q]\right|^{2}\right]} .
\end{aligned}
$$

From (1) and (2), the lowpass equivalent of the received signal $r_{\ell}(t)$ from the node $v$ through the link $\ell$ can be expressed as

$$
r_{\ell}(t)=e^{j 2 \pi \Delta f_{\ell} t+j \psi_{\ell}} \sum_{q=0}^{Q_{\ell}-1} h_{\ell}[q] u_{v}\left(t-q t_{\mathrm{s}}\right)+w(t)
$$

where $\Delta f_{\ell}$ denotes the frequency offset between two nodes involved in link $\ell, \psi_{\ell}$ denotes the initial phase difference between the two nodes, and $w(t)$ denotes a white Gaussian noise process with zero mean and autocorrelation function $E\left[w^{*}(t) w(t+\right.$ $\tau)]=\sigma^{2} \delta(\tau)$. When $r_{\ell}(t)$ is sampled at $t=n t_{\mathrm{s}}$, the received sample $r_{\ell}[n]$ can be expressed as

$$
\begin{aligned}
r_{\ell}[n] & \triangleq r_{\ell}\left(n t_{\mathrm{s}}\right) \\
& =e^{j \frac{2 \pi \varepsilon_{\ell} n}{N}+j \psi_{\ell}} \sum_{q=0}^{Q_{\ell}-1} h_{\ell}[q] u_{v}[n-q]+w[n]
\end{aligned}
$$

where $\varepsilon_{\ell}$ denotes the normalized frequency offset defined as $\varepsilon_{\ell} \triangleq \Delta f_{\ell} N t_{\mathrm{s}}$.

\section{Space-Time Cooperation Protocol}

In the CO-OFDM system, transmission of each frame involves two subsequent transmit phases: the listening phase and the cooperation phase, which are associated with the listening subframe and the cooperation subframe, respectively. Fig. 3 illustrates how wireless links are established during each phase. In the listening phase, the source broadcasts a listening subframe to the relay and destination without space-time coding. If the destination succeeds in decoding the listening subframe, the following cooperation phase is ignored at the destination. Otherwise, the destination attempts to decode the succeeding cooperation subframe. Note that the relay and destination can 
realize whether decoding of each subframe is successful or not by computing the checksum of the FCS.

In the cooperation phase, the source constructs and transmits a cooperation subframe, which corresponds to one half portion of the space-time-coded version of the listening subframe. The behavior of the relay depends on whether it has succeeded or not in decoding the preceding listening subframe. If the relay has succeeded in decoding, the relay also constructs and transmits a cooperation subframe, which corresponds to another half portion of the space-time-coded signal. Then, the destination may receive the complete space-time-coded signal from the source and relay and, thus, can decode the cooperation subframe more reliably than the listening subframe. Otherwise, if the relay has failed to decode the listening subframe, it is silent in the cooperation phase. Even in this situation, the destination may be able to decode the cooperation subframe from only the half portion of the space-time-coded signal from the source, if the space-time code is designed to deal with this case. We allow the listening and cooperation subframes to be transmitted at different transmission rates, which means that the length of the two subframes can be different. When the transmission rates of the listening and cooperation subframes are $\alpha_{1}$ and $\alpha_{2}$, respectively, the overall transmission rate $\alpha$ is computed as $\alpha=\alpha_{1} \alpha_{2} /\left(\alpha_{1}+\alpha_{2}\right)$. As a way of performance enhancement, in the cooperation phase, we combine the cooperation subframe and the preceding listening subframe at the destination. This combining is incorporated into the Viterbi decoder, by weighting each bit with the combined channel gain when computing path metrics. Note that the combining is possible even when the two phases are transmitted at different rates derived from code puncturing.

We adopt the Alamouti code to construct space-time-coded signals in the cooperation phase, since it provides full rate and full diversity with a simple decoding architecture [2]. Accordingly, each pair of OFDM symbols in a frame form a space-time coding block. Specifically, a block of space-time encoded transmit symbols for the data subcarrier $k \in \mathbb{I}_{d}$ in the $m$ th and $(m+1)$ th OFDM symbols can be expressed in matrix form as

$$
\left[\begin{array}{cc}
X_{S, m}[k] & X_{S, m+1}[k] \\
X_{R, m}[k] & X_{R, m+1}[k]
\end{array}\right]=\left[\begin{array}{cc}
D_{m}[k] & -D_{m+1}^{*}[k] \\
D_{m+1}[k] & D_{m}^{*}[k]
\end{array}\right]
$$

where $X_{v, m}[k]$ denotes the space-time encoded symbol transmitted from node $v$ over the data subcarrier $k$ in the $m$ th OFDM symbol, and $D_{m}[k]$ denotes the corresponding transmit symbol prior to space-time encoding. When both the source and relay transmit the cooperation subframe, the received symbols $R_{m}[k]$ and $R_{m+1}[k]$ at the destination can be arranged as

$$
\begin{array}{r}
{\left[\begin{array}{c}
R_{m}[k] \\
R_{m+1}^{*}[k]
\end{array}\right]=\left[\begin{array}{cc}
\mathcal{H}_{S D, m}[k] & \mathcal{H}_{R D, m}[k] \\
\mathcal{H}_{R D, m+1}^{*}[k] & -\mathcal{H}_{S D, m+1}^{*}[k]
\end{array}\right]\left[\begin{array}{c}
D_{m}[k] \\
D_{m+1}[k]
\end{array}\right]} \\
+\left[\begin{array}{c}
W_{m}[k] \\
W_{m+1}^{*}[k]
\end{array}\right]
\end{array}
$$

where $\mathcal{H}_{\ell, m}[k]$ denotes the frequency domain channel response of the link $\ell$ for the subcarrier $k$ during the $m$ th OFDM symbol, and $W_{m}[k]$ is the additive white Gaussian noise (AWGN) with zero mean and variance of $\sigma^{2}$. Note that the channel response $\mathcal{H}_{\ell, m}[k]$ reflects the transmit power $P_{v}$, the initial phase difference $\psi_{\ell}$, the effect of residual timing error $\bar{\nu}_{\ell}$, and that of residual frequency offset $\bar{\varepsilon}_{\ell}$ after synchronization, as well as the wireless channel in (2): $\mathcal{H}_{\ell, m}[k]$ is expressed as

$$
\begin{aligned}
\mathcal{H}_{\ell, m}[k]= & \frac{\sqrt{P_{v}}}{\sqrt{N}} \sum_{n=0}^{N-1} h_{\ell}\left[\left(n-\bar{\nu}_{\ell}\right) \bmod N\right] \\
& \times e^{j \frac{2 \pi \bar{\varepsilon}_{\ell}}{N}\left(n-\bar{\nu}_{\ell}+m L_{\mathrm{sym}}+L_{p}\right)+j \psi_{\ell}} e^{-j \frac{2 \pi k n}{N}} \\
= & e^{j \frac{2 \pi \bar{\varepsilon}_{\ell}}{N}\left(m L_{\mathrm{sym}}+L_{p}\right)+j \psi_{\ell}} \frac{\sqrt{P_{v}}}{\sqrt{N}} \sum_{n=0}^{N-1} \\
& \times h_{\ell}\left[\left(n-\bar{\nu}_{\ell}\right) \bmod N\right] e^{j \frac{2 \pi \bar{\varepsilon}_{\ell}\left(n-\bar{\nu}_{\ell}\right)}{N}} e^{-j \frac{2 \pi k n}{N}} \\
\approx & e^{j \frac{2 \pi \bar{\varepsilon}_{\ell}}{N}\left(m L_{\mathrm{sym}}+L_{p}+(N-1) / 2\right)-j \frac{2 \pi k \bar{\nu}_{\ell}}{N}}+j \psi_{\ell} \\
& \times \frac{\sqrt{P_{v}} \sin \left(\pi \bar{\varepsilon}_{\ell}\right)}{N \sin \left(\pi \bar{\varepsilon}_{\ell} / N\right)} \frac{1}{\sqrt{N}} \sum_{n=0}^{Q_{\ell}-1} h_{\ell}[n] e^{-j \frac{2 \pi k n}{N}} \\
= & e^{j \frac{2 \pi \bar{\varepsilon}_{\ell}}{N}(m+1) L_{\mathrm{sym}}} H_{\ell}[k] \\
H_{\ell}[k] \triangleq & e^{j \frac{2 \pi \bar{\varepsilon}_{\ell}}{N}\left(L_{p}-L_{\mathrm{sym}}+(N-1) / 2\right)-j \frac{2 \pi k \bar{\nu}_{\ell}}{N}+j \psi_{\ell}} \\
& \times \frac{\sqrt{P_{v}} \sin \left(\pi \bar{\varepsilon}_{\ell}\right)}{N \sin \left(\pi \bar{\varepsilon}_{\ell} / N\right)} \frac{1}{\sqrt{N}} \sum_{n=0}^{Q_{\ell}-1} h_{\ell}[n] e^{-j \frac{2 \pi k n}{N}}
\end{aligned}
$$

where $H_{\ell}[k]$ denotes the channel response in the last period of the channel estimation sequence, $h_{\ell}[n]=0$ for $n \geq Q_{\ell}$, and the approximation holds when $\bar{\varepsilon}_{\ell}$ is sufficiently small such that $e^{j 2 \pi \bar{\varepsilon}_{\ell}} \approx 1$. For decoding of the space-time-coded symbols, the destination assumes $\mathcal{H}_{\ell, m+1}[k]=\mathcal{H}_{\ell, m}[k]$, which is reasonable if $e^{j 2 \pi \bar{\varepsilon}_{\ell} L_{\mathrm{sym}} / N} \approx 1$. If channel estimates $\hat{\mathcal{H}}_{\ell, m}[k]$, $\ell=S D, R D$ are available, the destination can obtain soft decisions on $D_{m}[k]$ and $D_{m+1}[k]$ using the maximum likelihood decoding rule as [2]

$$
\begin{array}{r}
{\left[\begin{array}{c}
\hat{D}_{m}[k] \\
\hat{D}_{m+1}[k]
\end{array}\right]=\frac{1}{\hat{\Lambda}_{m}[k]}\left[\begin{array}{cc}
\hat{\mathcal{H}}_{S D, m}^{*}[k] & \hat{\mathcal{H}}_{R D, m}[k] \\
\hat{\mathcal{H}}_{R D, m}^{*}[k] & -\hat{\mathcal{H}}_{S D, m}[k]
\end{array}\right]} \\
\times\left[\begin{array}{c}
R_{m}[k] \\
R_{m+1}^{*}[k]
\end{array}\right] .
\end{array}
$$

where $\hat{\Lambda}_{m}[k] \triangleq\left|\hat{\mathcal{H}}_{S D, m}[k]\right|^{2}+\left|\hat{\mathcal{H}}_{R D, m}[k]\right|^{2}$. In the special case when the relay transmits nothing in the cooperation phase, the signal components associated with the relay will not appear in the received symbol vector in (7). In this case, the channel estimates $\hat{\mathcal{H}}_{R D, m}[k]$ at the destination may be very small, ideally becoming zero. Thus, even though the destination does not realize that the relay is silent, the decoding rule in (9) still works. It should be noted that the Alamouti code can alternatively be implemented in the form of a space-frequency coding instead of a space-time coding, if the channel variation is negligible over two adjacent subcarriers.

One implementation issue to be pointed out is the decoding latency problem. In practice, there may exist nonnegligible latency in decoding listening subframes at the relay. This means that the relay cannot transmit the cooperation subframe right 
after receiving the listening subframe. Therefore, if the source transmits the listening and cooperation subframes successively, the transmit signals from the source and relay may not be synchronized. We solve this problem by forcing the source to intentionally delay transmissions of the cooperation subframe, so that the source and relay can synchronize their transmissions. By reordering and interlacing the listening and cooperation subframes, we can minimize the waste of bandwidth due to this delayed transmission. In addition, we may need to insert a short period of silence between consecutive listening and cooperation subframes, when the relay cannot transmit and receive at the same time due to the half-duplex constraint [7], [8]. The duration of each period should be greater than the propagation delay between the source and relay. The details of the decoding latency and propagation delay issues can be found in [23].

\section{TIMING AND FREQUENCY SYNCHRONIZATION}

Timing and frequency synchronization should be established prior to channel estimation and data demodulation. We propose algorithms that utilize the repetitive structure of the synchronization signals in the preamble described in Section II-B. In the listening phase, we consider only the $S D$ link, since the same algorithm is applied to the $S R$ link. From (5), the received signal samples at the destination in each phase are given as

$$
r[n]=\left\{\begin{array}{c}
e^{j \frac{2 \pi \varepsilon_{S} D^{n}}{N}+j \psi_{S D} \sum_{q=0}^{Q_{S D}-1} h_{S D}[q] u_{S}[n-q]+w[n]}, \\
\text { listening phase } \\
e^{j \frac{2 \pi \varepsilon_{S} D^{n}}{N}+j \psi_{S D} \sum_{q=0}^{Q_{S D}-1} h_{S D}[q] u_{S}[n-q]} \\
+e^{j \frac{2 \pi \varepsilon_{R D^{n}}}{N}}+j \psi_{R D} \sum_{q=0}^{Q_{R D}-1} h_{R D}[q] u_{R}[n-q-\zeta] \\
+w[n], \quad \text { cooperation phase }
\end{array}\right.
$$

where $\zeta$ denotes the timing offset between the received signals from the source and relay. We assume that the source and relay are roughly synchronized by an external clock so that the timing offset at the destination is not greater than the length of the cyclic prefix, i.e., $|\zeta| \leq L_{\mathrm{cp}}$.

\section{A. Timing Synchronization}

The proposed timing synchronization algorithm is divided into three sequential stages: coarse timing synchronization, fine timing synchronization, and frame synchronization, each of which is described as follows.

Coarse timing synchronization is responsible for making the receiver realize that there exist significant incoming signals. For this purpose, we measure the moving average of the received signal power as

$$
\phi_{\mathrm{ct}}[\mu]=\sum_{n=0}^{L_{m}-1}|r[\mu-n]|^{2}
$$

where $L_{m}$ denotes the moving average window size in samples. Note that computations for the moving averages in (11) can be simplified using a recursive implementation as

$$
\phi_{\mathrm{ct}}[\mu]=\phi_{\mathrm{ct}}[\mu-1]-\left|r\left[\mu-L_{m}\right]\right|^{2}+|r[\mu]|^{2} .
$$

The power estimate $\phi_{\mathrm{ct}}[\mu]$ is compared with a threshold $\Gamma$ every sampling interval until $\phi_{\mathrm{ct}}\left[\mu^{\prime}\right]>\Gamma$, at which point, a coarse timing is declared as $\hat{\mu}_{\mathrm{ct}}=\mu^{\prime}$. The threshold $\Gamma$ is set according to the constant-false-alarm-rate criterion. In the absence of signal, since $\phi_{\mathrm{ct}}[\mu]$ is the sum of squares of $L_{m}$ AWGN samples, it follows the central chi-square distribution with variance parameter $\sigma^{2} / 2$ and $2 L_{m}$ degrees of freedom [20]. Using the cumulative distribution function of the central chi-square distribution, we can determine the threshold $\Gamma$ as

$$
\Gamma=\frac{\hat{\sigma}^{2}}{2}\left(Q_{L_{m}}^{-1}\left(0, P_{f}\right)\right)^{2}
$$

where $\hat{\sigma}^{2}$ denotes an estimate of the noise power at the destination, $P_{f}$ denotes the target probability of false alarm, and $Q_{L_{m}}^{-1}\left(0, P_{f}\right)$ represents the inverse of the Marcum $Q$-function $Q_{L_{m}}(0, \cdot)$ [20]. It should be noted that the coarse timing synchronization stage needs to be executed only in the initial link setup. Once a link is initialized, we can skip this stage and go to the following fine timing synchronization stage.

Fine timing synchronization detects one of the boundaries of the repeated synchronization sequence. We use a squared cross correlation between the received samples $r[n]$ and local synchronization sequence $s_{v}[n]$, as in the code acquisition of $\mathrm{SS}$ signals [24]. A filter matched to a synchronization sequence $s_{v}[n]$ can produce cross correlations for $L_{\text {sync }}$ candidates of fine timing from the received samples $r[n], \hat{\mu}_{\mathrm{ct}}+1 \leq n<$ $\hat{\mu}_{\text {ct }}+2 L_{\text {sync }}$, where the front $L_{\text {sync }}-1$ samples are used to fill the memory elements of the filter. The resulting squared cross correlations in the listening phase can be written as

$$
\begin{aligned}
& \theta[\mu]=\left|\sum_{n=0}^{L_{\mathrm{sync}}-1} s_{S}^{*}\left[L_{\mathrm{sync}}-1-n\right] r[\mu-n]\right|^{2} \\
& \hat{\mu}_{\mathrm{ct}}+L_{\mathrm{sync}} \leq \mu<\hat{\mu}_{\mathrm{ct}}+2 L_{\mathrm{sync}} .
\end{aligned}
$$

In the cooperation phase, we combine the squared cross correlations for the sequences $s_{S}[n]$ and $s_{R}[n]$. The combined squared cross correlations are given as

$$
\begin{aligned}
& \theta[\mu]=\left|\sum_{n=0}^{L_{\mathrm{sync}}-1} s_{S}^{*}\left[L_{\mathrm{sync}}-1-n\right] r[\mu-n]\right|^{2} \\
&+\left|\sum_{n=0}^{L_{\mathrm{sync}}-1} s_{R}^{*}\left[L_{\mathrm{sync}}-1-n\right] r[\mu-n]\right|^{2} \\
& \hat{\mu}_{\mathrm{ct}}+L_{\mathrm{sync}} \leq \mu<\hat{\mu}_{\mathrm{ct}}+2 L_{\mathrm{sync}} .
\end{aligned}
$$

When there are multiple significant paths in the channel, or when the timing offset $\zeta$ in (10) is nonzero in the cooperation phase, the squared cross correlations in (14) and (15) exhibit multiple peaks. In this case, decisions based on (14) and (15) may yield a timing that causes a great loss of signal energy. It is desirable to determine a fine timing that maximizes the total signal energy captured in the FFT window for channel estimation and data demodulation. To this end, we propose to 
combine adjacent $K$ squared cross correlation values in (14) and (15) as

$$
\begin{array}{r}
\phi_{\mathrm{ft}}[\mu]=\sum_{j=0}^{K-1} \theta\left[\hat{\mu}_{\mathrm{ct}}+L_{\mathrm{sync}}+\left(\left(\mu-\hat{\mu}_{\mathrm{ct}}+j\right) \bmod L_{\mathrm{sync}}\right)\right] \\
\hat{\mu}_{\mathrm{ct}}+L_{\mathrm{sync}} \leq \mu<\hat{\mu}_{\mathrm{ct}}+2 L_{\mathrm{sync}} .
\end{array}
$$

Based on (16), the fine timing $\hat{\mu}_{\mathrm{ft}}$ is determined as

$$
\hat{\mu}_{\mathrm{ft}}=\underset{\hat{\mu}_{\mathrm{ct}}+L_{\mathrm{sync}} \leq \mu<\hat{\mu}_{\mathrm{ct}}+2 L_{\mathrm{sync}}}{\operatorname{argmax}} \phi_{\mathrm{ft}}[\mu] .
$$

Frame synchronization is the final stage of timing synchronization that determines the frame boundary or the starting point of the channel estimation signals. We utilize the fact that the last period of the synchronization sequence is the negative of the previous periods, as explained in Section II-B. Starting from the position of the fine timing estimate, we compute autocorrelations $\gamma[n]$ between each of two consecutive periods of the received samples as

$$
\begin{aligned}
\gamma[n]=\sum_{j=0}^{L_{\mathrm{sync}}-1} & r^{*}\left[j+\hat{\mu}_{\mathrm{ft}}+n L_{\mathrm{sync}}\right] \\
& \times r\left[j+\hat{\mu}_{\mathrm{ft}}+(n+1) L_{\mathrm{sync}}\right], \quad n \geq 0 .
\end{aligned}
$$

We note that when the autocorrelation is taken between the last two periods of the synchronization sequence, the phase of $\gamma[n]$ may ideally change by $\pi$ due to different signs of the signals during the two periods. The destination can detect the point of this phase change by examining

$$
\phi_{\text {fr }}[n]=\operatorname{Re}\left\{\gamma^{*}[n] \gamma[n+1]\right\}, \quad n \geq 0
$$

where $\operatorname{Re}(\cdot)$ denotes the real component of a complex number. If $\phi_{f r}\left[n^{\prime}\right]<0$ for a certain $n^{\prime} \geq 0$, an estimate $\hat{\mu}_{f r}$ of frame timing is found as

$$
\hat{\mu}_{f r}=\hat{\mu}_{\mathrm{ft}}+\left(n^{\prime}+3\right) L_{\mathrm{sync}}
$$

which completes the timing synchronization. The resulting residual timing errors for the $S D$ link and $R D$ link are given as

$$
\begin{aligned}
& \bar{\nu}_{S D}=N_{\mathrm{sync}} L_{\mathrm{sync}}-\hat{\mu}_{f r} \\
& \bar{\nu}_{R D}=N_{\mathrm{sync}} L_{\mathrm{sync}}+\zeta-\hat{\mu}_{f r} .
\end{aligned}
$$

\section{B. Frequency Offset Estimation and Correction}

We utilize the two-phase structure of the cooperation protocol in Section II-D to estimate and correct the frequency offsets. We first derive an estimation algorithm that works between transmitter/receiver pair. Then, we discuss how this estimation algorithm can be applied to synchronize carrier frequencies of the three nodes.

The estimation algorithm reuses the autocorrelation in (18) similarly to the scheme in [19]. If the fine timing synchroniza- tion is correct, for the received signals from the link $\ell$, it can be shown that

$$
\begin{array}{r}
\gamma[n]=e^{j 2 \pi \Delta f_{\ell} L_{\mathrm{sync}} t_{\mathrm{s}}} \times \sum_{j=0}^{L_{\mathrm{sync}}-1}\left|\sum_{q=0}^{Q_{\ell}-1} h_{\ell}[q] u_{v}\left[j+\hat{\mu}_{\mathrm{ft}}-q\right]\right|^{2} \\
+\eta[n], \quad 0 \leq n \leq n^{\prime}
\end{array}
$$

where $\eta[n]$ is the noise component. If the frequency offset for the link $\ell$ satisfies $\left|\Delta f_{\ell}\right| \leq 1 /\left(2 L_{\text {sync }} t_{\mathrm{s}}\right)$, which is necessary to avoid $2 \pi n$ phase ambiguities, the frequency offset $\Delta f_{\ell}$ can be estimated as

$$
\Delta \hat{f}_{\ell}=\frac{1}{2 \pi L_{\text {sync }} t_{\mathrm{s}}} \measuredangle\left\{\sum_{n=0}^{n^{\prime}-1} \gamma[n]\right\}
$$

where $\measuredangle\{\cdot\}$ represents the phase of a complex number. In (23), we do not use $\gamma\left[n^{\prime}\right]$ to protect the estimate $\Delta \hat{f}_{\ell}$ from being affected by the last period of synchronization sequence due to possible timing error.

The above estimation algorithm is used in conjunction with the cooperation protocol to correct the frequency offsets among the three nodes. Basically, we regard the oscillator frequency $f_{S}$ of the source as the reference and make the relay and destination estimate and adjust their frequencies $f_{R}$ and $f_{D}$ as close to $f_{S}$ as possible. First, in the listening phase, the relay estimates the frequency offset between the source and relay, $\Delta f_{S R}=f_{S}$ $f_{R}$, and the destination estimates that between the source and destination $\Delta f_{S D}=f_{S}-f_{D}$, respectively, using the above algorithm. Using the estimates $\Delta \hat{f}_{S R}$ and $\Delta \hat{f}_{S D}$, the relay and destination, respectively, adjust their carrier frequencies as

$$
\begin{aligned}
& f_{R}^{\prime}=f_{R}+\Delta \hat{f}_{S R} \\
& f_{D}^{\prime}=f_{D}+\Delta \hat{f}_{S D} .
\end{aligned}
$$

This procedure may make the frequencies of the three nodes synchronized to some extent. To further reduce the residual offsets, we can also use the cooperation phase. However, in the cooperation phase, the destination cannot distinguish between the signal from the source and that from the relay. Nevertheless, the destination can estimate the offset $\Delta f_{S R, D}^{\prime}$ between the frequency of destination $f_{D}^{\prime}$ and that of the composite signals it sees and adjust its frequency as

$$
f_{D}^{\prime \prime}=f_{D}^{\prime}+\Delta \hat{f}_{S R, D}^{\prime}
$$

After correction, the residual normalized frequency offset for each link is given as

$$
\begin{aligned}
& \bar{\varepsilon}_{S D}=\left(f_{S}-f_{D}^{\prime \prime}\right) N t_{\mathrm{s}} \\
& \bar{\varepsilon}_{S R}=\left(f_{S}-f_{R}^{\prime}\right) N t_{\mathrm{s}} \\
& \bar{\varepsilon}_{R D}=\left(f_{R}^{\prime}-f_{D}^{\prime \prime}\right) N t_{\mathrm{s}} .
\end{aligned}
$$

It should be noted that in the listening phase, the estimate $\Delta \hat{f}_{S R}$ may be more accurate than $\Delta \hat{f}_{S D}$, if the relay is closer to the source than the destination is, which is usually the case. This is simply because the relay will receive stronger signal from the source than the destination when the relay is closer to the 
source. Hence, the error between $f_{S}$ and $f_{R}^{\prime}$ is expected to be smaller than the error between $f_{S}$ and $f_{D}^{\prime}$ after the listening phase, and thus, the second correction step in (25) is expected to improve the performance. A remarkable merit of the proposed correction scheme is that it does not need any feedback signal, since receiving nodes attempt to lock their frequencies to that of the transmit node.

\section{Channel Estimation}

In this section, we propose a channel estimation algorithm for CO-OFDM systems. We present our algorithm only for the cooperation phase, since the algorithm can easily be simplified to an algorithm for the listening phase. The initial estimation is based on the channel estimation signals in the preamble, whereas the phase tracking is based on the pilot subcarriers inserted in each OFDM symbol.

\section{A. Initial Channel Estimation}

The initial channel estimation algorithm uses the $N_{\text {ce }}$ repetitions of the channel estimation sequence $\left\{C_{v}[k]\right\}$ and works in the frequency domain after applying an FFT to the received signals. In the cooperation phase, the received channel estimation signals at the destination can be expressed as

$$
\begin{array}{r}
R_{m}[k]=\mathcal{H}_{S D,-m}[k] C_{S}[k]+\mathcal{H}_{R D,-m}[k] C_{R}[k]+W_{m}[k] \\
0 \leq m<N_{\mathrm{ce}}, \quad k \in \mathbb{I}_{u}
\end{array}
$$

where $\mathcal{H}_{\ell,-m}[k]$ represents the frequency domain channel response for the subcarrier $k$ and link $\ell$ in the $\left(N_{\mathrm{ce}}-m\right)$ th period of the channel estimation sequence and can be expressed as (8). The destination needs to estimate two sets of distinct channels, $\mathcal{H}_{S D, m}[k]$ and $\mathcal{H}_{R D, m}[k]$. For each period of signals, we first multiply a normalized conjugate of each channel estimation sequence to get rough channel estimates as

$$
\begin{aligned}
\mathcal{H}_{S D,-m}^{\prime}[k]= & \frac{R_{m}[k] C_{S}^{*}[k]}{\left|C_{S}[k]\right|^{2}} \\
= & \mathcal{H}_{S D,-m}[k]+\frac{\mathcal{H}_{R D,-m}[k] C_{R}[k] C_{S}^{*}[k]}{\left|C_{S}[k]\right|^{2}} \\
& +\frac{W_{m}[k] C_{S}^{*}[k]}{\left|C_{S}[k]\right|^{2}} \\
\mathcal{H}_{R D,-m}^{\prime}[k]= & \frac{R_{m}[k] C_{R}^{*}[k]}{\left|C_{R}[k]\right|^{2}} \\
= & \mathcal{H}_{R D,-m}[k]+\frac{\mathcal{H}_{S D,-m}[k] C_{S}[k] C_{R}^{*}[k]}{\left|C_{R}[k]\right|^{2}} \\
& +\frac{W_{m}[k] C_{R}^{*}[k]}{\left|C_{R}[k]\right|^{2}} .
\end{aligned}
$$

From the second terms on the right-hand side of (28), we can see that the estimates suffer from interference from each other. In order to suppress the interference, we exploit the proposed structure of sequences $\left\{C_{S}[k]\right\}$ and $\left\{C_{R}[k]\right\}$ described in Section II-B: $\left\{C_{S}[k]\right\}$ and $\left\{C_{R}[k]\right\}$ are orthogonal over each pair of adjacent subcarriers. Specifically, we suppress the interference by averaging $\mathcal{H}_{S D,-m}^{\prime}[k]$ and $\mathcal{H}_{R D,-m}^{\prime}[k]$ over each pair of adjacent subcarriers, assuming that the channel variation is minimal over each pair of subcarriers. The averaged channel responses are given as

$$
\begin{array}{r}
\mathcal{H}_{\ell, m}^{\prime}[\kappa]=\left(\mathcal{H}_{\ell, m}^{\prime}[\kappa-0.5]+\mathcal{H}_{\ell, m}^{\prime}[\kappa+0.5]\right) / 2 \\
\kappa \in \mathbb{I}_{u}^{\prime}, \ell=S D, R D
\end{array}
$$

where $\mathbb{I}_{u}^{\prime}=\left\{-N_{u} / 2+0.5, \ldots,-1.5,1.5, \ldots, N_{u} / 2-0.5\right\}$ is a set of indexes and each index is the mean of each pair of adjacent indexes in $\mathbb{I}_{u}$. Note that these estimates reflect phase rotations due to residual frequency offset after frequency correction. We regard the last period of the channel estimation sequence as the time reference of the initial channel estimation and align the phases of channel responses in (29) to those of the last period as

$$
\begin{array}{r}
\mathcal{H}_{\ell,-m}^{\prime \prime}[\kappa]=\mathcal{H}_{\ell,-m}^{\prime}[\kappa] e^{j \measuredangle\left\{\sum_{\kappa \in \mathbb{H}_{u}^{\prime}} \mathcal{H}_{\ell,-m}^{\prime *}[\kappa] \mathcal{H}_{\ell, 0}^{\prime}[\kappa]\right\}} \\
0 \leq m<N_{\mathrm{ce}} .
\end{array}
$$

Then, we average the estimates in (30) over $N_{\text {ce }}$ periods to get

$$
\tilde{H}_{\ell}[\kappa]=\frac{1}{N_{\mathrm{ce}}} \sum_{m=0}^{N_{\mathrm{ce}}} \mathcal{H}_{\ell,-m}^{\prime \prime}[\kappa], \quad \kappa \in \mathbb{I}_{u}^{\prime}, \quad \ell=S D, R D .
$$

Note that (31) provides only partial channel responses every two subcarriers due to the averaging step in (29). To obtain full channel responses for all data and pilot subcarriers, we interpolate the partial responses as

$$
\left\{\tilde{H}_{\ell}[k]: k \in \mathbb{I}_{u}\right\}=I\left(\left\{\tilde{H}_{\ell}[\kappa]: \kappa \in \mathbb{I}_{u}^{\prime}\right\}\right), \quad \ell=S D, R D
$$

where $I(\cdot)$ denotes an interpolation function.

The estimates in (32) may be noisy particularly if $N_{\text {ce }}$ is small. To suppress noise and residual interference, we apply a lowpass filter to the estimates in (32), as in [25]. Let $\tilde{\mathbf{H}}_{\ell} \triangleq$ $\left[\tilde{H}_{\ell}\left[-N_{u} / 2\right] \cdots \tilde{H}_{\ell}\left[N_{u} / 2\right]\right]^{\mathrm{T}}$. Then, filtered time domain channel responses $\hat{\mathbf{h}}_{\ell}$ can be obtained as

$$
\begin{aligned}
& \hat{\mathbf{h}}_{\ell} \triangleq\left[\begin{array}{llll}
\hat{h}_{\ell}[0] & \hat{h}_{\ell}[1] & \cdots & \hat{h}_{\ell}[M-1
\end{array}\right]^{\mathrm{T}} \\
& =\left(\mathbf{F}^{\mathrm{H}} \mathbf{F}\right)^{-1} \mathbf{F}^{\mathrm{H}} \tilde{\mathbf{H}}_{\ell}, \quad \ell=S D, S R, R D
\end{aligned}
$$

where $M$ is a parameter that represents an expected length of $\hat{\mathbf{h}}_{\ell}$, and the elements of a partial discrete Fourier transform matrix $\mathbf{F}$ are given as $[\mathbf{F}]_{k, \ell}=e^{j \pi(\ell-1)\left(N_{u}-2 k+2\right) / N}$ and $[\mathbf{F}]_{k+N_{u} / 2, \ell}=e^{j \pi(\ell-1)(-2 k) / N}$ for $1 \leq k \leq N_{u} / 2,1 \leq$ $\ell \leq M$. From (33), initial channel estimates are computed as

$$
\begin{aligned}
\hat{\mathbf{H}}_{\ell} & \triangleq\left[\begin{array}{lll}
\hat{H}_{\ell}\left[-N_{u} / 2\right] & \cdots & \hat{H}_{\ell}\left[N_{u} / 2\right]
\end{array}\right]^{\mathrm{T}} \\
& =\mathbf{F} \hat{\mathbf{h}}_{\ell}, \quad \ell=S D, S R, R D .
\end{aligned}
$$




\section{B. Phase Tracking}

Although we assume that the channel does not vary during each frame, phase rotation due to residual frequency offset should be tracked and reflected in the channel estimates every symbol or space-time coding block. This is because the phase rotation accumulates over time as shown in (8), although it may be negligible during each OFDM symbol. We use the received signals from pilot subcarriers to track this phase rotation. In the cooperation phase, the phase rotation can be estimated every space-time coding block or every two OFDM symbols once we neglect conjugate operations when constructing space-time coding blocks for pilot subcarriers as in (6).

Assuming that the phase rotations are negligible over two OFDM symbols, the received signals for the pilot subcarriers during the $m$ th and $(m+1)$ th OFDM symbols can be expressed as

$$
\begin{aligned}
R_{m}[k] & =\left(H_{S D}[k] e^{j \varphi_{S D, m}}+H_{R D}[k] e^{j \varphi_{R D, m}}\right) P[k] \\
R_{m+1}[k] & =\left(-H_{S D}[k] e^{j \varphi_{S D, m+1}}+H_{R D}[k] e^{j \varphi_{R D}, m+1}\right) P[k] \\
& \approx\left(-H_{S D}[k] e^{j \varphi_{S D, m}}+H_{R D}[k] e^{j \varphi_{R D, m}}\right) P[k]
\end{aligned}
$$

where $\varphi_{\ell, m}=\left(2 \pi \bar{\varepsilon}_{\ell} / N\right)(m+1) L_{\text {sym }}$ denotes the amount of phase rotation of the channel in the $m$ th OFDM symbol from the point of initial channel estimation, due to the residual frequency offset $\bar{\varepsilon}_{\ell}$, and $P[k]$ is the pilot symbol for the pilot subcarrier $k$. The subtraction and addition of (35) and (36) result in

$$
\begin{aligned}
& R_{m}[k]-R_{m+1}[k] \approx 2 H_{S D}[k] e^{j \varphi_{S D, m}} P[k] \\
& R_{m}[k]+R_{m+1}[k] \approx 2 H_{R D}[k] e^{j \varphi_{R D}, m} P[k] .
\end{aligned}
$$

Therefore, the phase rotations can be estimated as

$$
\begin{aligned}
& \hat{\varphi}_{S D, m}=\measuredangle\left\{\sum_{k \in \mathbb{\Pi}_{p}}\left(R_{m}[k]-R_{m+1}[k]\right) \hat{H}_{S D}^{*}[k] P^{*}[k]\right\} \\
& \hat{\varphi}_{R D, m}=\measuredangle\left\{\sum_{k \in \mathbb{I}_{p}}\left(R_{m}[k]+R_{m+1}[k]\right) \hat{H}_{R D}^{*}[k] P^{*}[k]\right\} .
\end{aligned}
$$

From (34), (39), and (40), we compute channel estimates for the $m$ th and $(m+1)$ th OFDM symbols as

$$
\hat{\mathcal{H}}_{\ell, m}[k]=\hat{\mathcal{H}}_{\ell, m+1}[k]=\hat{H}_{\ell}[k] e^{j \hat{\varphi}_{\ell, m}}, \quad \ell=S D, R D .
$$

\section{Performance Evaluation}

In this section, we evaluate the performance of the $\mathrm{CO}$ OFDM system by simulations. The main parameters of the CO-OFDM system used in simulations are summarized in Table I. Fig. 4 depicts how 48 data subcarriers, four pilot subcarriers, and 12 guard or null subcarriers are allocated
TABLE I

MAIN PARAMETERS OF THE CO-OFDM SyStem

\begin{tabular}{l|l}
\hline Parameter & Value \\
\hline \hline Total bandwidth $(W)$ & $4.625 \mathrm{MHz}$ \\
\hline Subcarrier frequency spacing & $72.27 \mathrm{kHz}$ \\
\hline Total number of subcarriers $(N)$ & 64 \\
\hline Number of data subcarriers $\left(N_{d}\right)$ & 48 \\
\hline Number of pilot subcarriers $\left(N_{p}\right)$ & 4 \\
\hline Number of guard or null subcarriers $\left(N_{g}\right)$ & 12 \\
\hline Length of cyclic prefix $\left(L_{c p}\right)$ & $10 \mathrm{samples}$ \\
\hline Sample duration $\left(t_{s}\right)$ & $0.216 \mu \mathrm{sec}$ \\
\hline OFDM symbol duration $\left(L_{s y m} t_{s}\right)$ & $16 \mu \mathrm{sec}$ \\
\hline Carrier frequency & $900 \mathrm{MHz}$ \\
\hline Moving average window size $L_{m}$ in $(11)$ & 63 \\
\hline Cross-correlation combining window size $K$ in $(16)$ & 6 \\
\hline Expected channel length $M$ in $(33)$ & 6 \\
\hline
\end{tabular}

to the frequency subchannels. For multipath fading models, we use the two channel models in [26], referred to as channel $\mathrm{A}$ and channel $\mathrm{B}$. The normalized multipath intensity profiles, $\left\{E\left[\left|h_{\ell}[q]\right|^{2}\right] / E\left[\sum_{j=0}^{Q_{\ell}-1}\left|h_{\ell}[j]\right|^{2}\right]: 0 \leq q<Q_{\ell}\right\}$, of the channel A and channel B are given by $\{0.9981,0.0019\}$ and $\{0.9410,0.0573,0,0.0017\}$, respectively. Every path gain $h_{\ell}[q]$ of the channel is assumed to follow an independent complex Gaussian distribution. We use a pair of Gold sequences with length $L_{\text {sync }}=63$ as synchronization sequences $\left\{s_{S}[n]\right\}$ and $\left\{s_{R}[n]\right\}$, since they have good autocorrelation and cross correlation properties [24]. For the channel estimation sequence $\left\{C_{S}[k]\right\}$ of the source, we employ the same binary sequence with length $N_{u}=52$ as in the IEEE 802.11a standard [15]. The channel estimation sequence $\left\{C_{R}[k]\right\}$ of the relay is constructed as explained in Section II-B. The periods of synchronization and channel estimation sequences are taken to be $N_{\text {sync }}=8$ and $N_{\text {ce }}=2$. We use the same pilot symbols as in the IEEE 802.11a standard [15]. In (13), the noise power estimate $\hat{\sigma}^{2}$ of the destination is assumed to be perfect, and the target probability of false alarm $P_{f}$ is set to $10^{-5}$.

We compare the performance of the proposed CO-OFDM system with that of two baseline systems without cooperation: a single antenna (SA) OFDM system and an Alamouti coded double antenna (DA) OFDM system, which we refer to as SA-OFDM and DA-OFDM, respectively. The SA-OFDM and DA-OFDM systems are the same as the CO-OFDM system except that the SA-OFDM system employs one transmit antenna without relay, and the DA-OFDM system employs two transmit antennas without relay. For the SA-OFDM and DAOFDM systems, we use a rate 1/3 convolutional code generated by polynomials $\left(133_{8}, 165_{8}, 171_{8}\right)$. In both the listening and cooperation phases of the CO-OFDM system, we use a rate $3 / 4$ convolutional code, which is obtained by puncturing the $1 / 3$ code. The puncturing pattern is [100100011], which means that only the first, fourth, eight, and ninth bits among a block of nine coded bits from the $1 / 3$ code are transmitted. The header part is always encoded using the rate $1 / 3$ code for all three systems. The interleaver size is chosen to be $n_{\text {row }}=16$ and $n_{\text {col }}=12$, which corresponds to one space-time coding block. Quaternary phase-shift keying is used as the data modulation scheme. It is assumed that the listening phase consumes $1 / 3$ of the total 


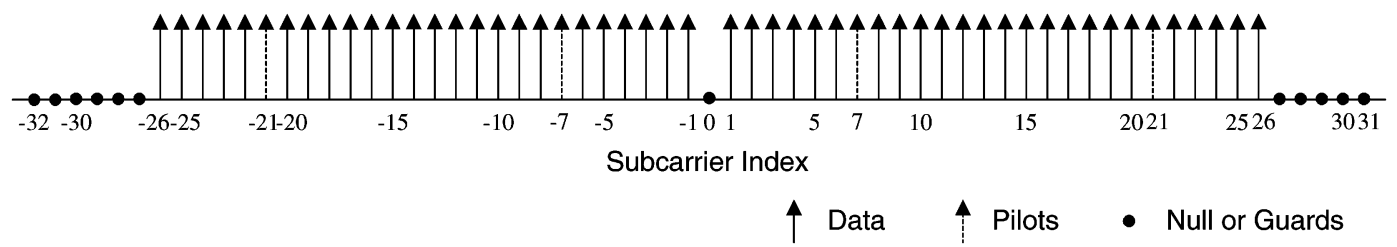

Fig. 4. Subcarrier allocation of the CO-OFDM system.

transmit power, and the source and relay transmit the same power in the cooperation phase: $P_{S}=P_{R}$. Correspondingly, the received $E_{b} / N_{0}$ is defined as

$$
E_{b} / N_{0}=\frac{2 \beta_{1}+\beta_{2}}{2 \beta_{1} \beta_{2}} \frac{P_{S}}{\sigma^{2}} E\left[\sum_{q=0}^{Q_{S D}}\left|h_{S D}[q]\right|^{2}\right]
$$

where $\beta_{1}$ and $\beta_{2}$ denote the code rate in the listening phase and cooperation phase, respectively. The decoding depth of the Viterbi decoder is fixed to 50 bits. With the parameters in Table I and under the above assumptions, the transmission rate of the CO-OFDM system is $2250 \mathrm{~kb} / \mathrm{s}$, and that of the SAOFDM and DA-OFDM systems is $2000 \mathrm{~kb} / \mathrm{s}$. The payload size of each frame is fixed to 256 bytes. For this payload size, taking all the overheads into account, the effective data rates reduce to $1572 \mathrm{kbps}$ for the CO-OFDM system, and $1668 \mathrm{kbps}$ for the SA-OFDM and DA-OFDM systems.

We first evaluate the performance of the proposed timing and frequency synchronization algorithms. In simulations, it is assumed that the timing offset $\zeta$ between the source and relay is an integer value uniformly distributed over $[-3,3]$ samples and that the initial frequency error of each node is assumed to be uniformly distributed over $[-2.0,2.0] \mathrm{ppm}$, which corresponds to $[-1.8,1.8] \mathrm{kHz}$ at the carrier frequency of $900 \mathrm{MHz}$. The geometric gains are assumed to be $G_{S R}=G_{R D}=0 \mathrm{~dB}$. Fig. 5 shows the probability of overall timing detection in the cooperation phase. We assume that timing synchronization is successful if either the strongest path in the $S D$ link or that in the $R D$ link has a timing error not greater than the length of cyclic prefix. The figure shows that probability of timing detection for fading channels is close to one, as long as the received SNR is greater than $10 \mathrm{~dB}$. For comparison purposes, in Fig. 5, we provide a plot for channel B with the combining window size $K$ equal to 1 . As expected, the use of the proposed cross correlation combining scheme is shown to be useful in increasing the detection probability.

Fig. 6 shows the sum of normalized residual frequency errors after frequency synchronization, which is defined to be $\left|\bar{\varepsilon}_{S D}\right|+\left|\bar{\varepsilon}_{R D}\right|$ with $\bar{\varepsilon}_{S D}$ and $\bar{\varepsilon}_{R D}$ given in (26), when the timing synchronization is correct. To show the effectiveness of the second correction stage in (25), we also present the sum of normalized residual frequency errors right after the listening phase. The results confirm that the second correction stage significantly decreases the residual frequency offset. Plots associated with the geometric gain $G_{S R}=0 \mathrm{~dB}$ and $10 \mathrm{~dB}$ for channel $\mathrm{B}$ verify that the second correction stage is more effective for higher $G_{S R}$, as discussed in Section III-B.

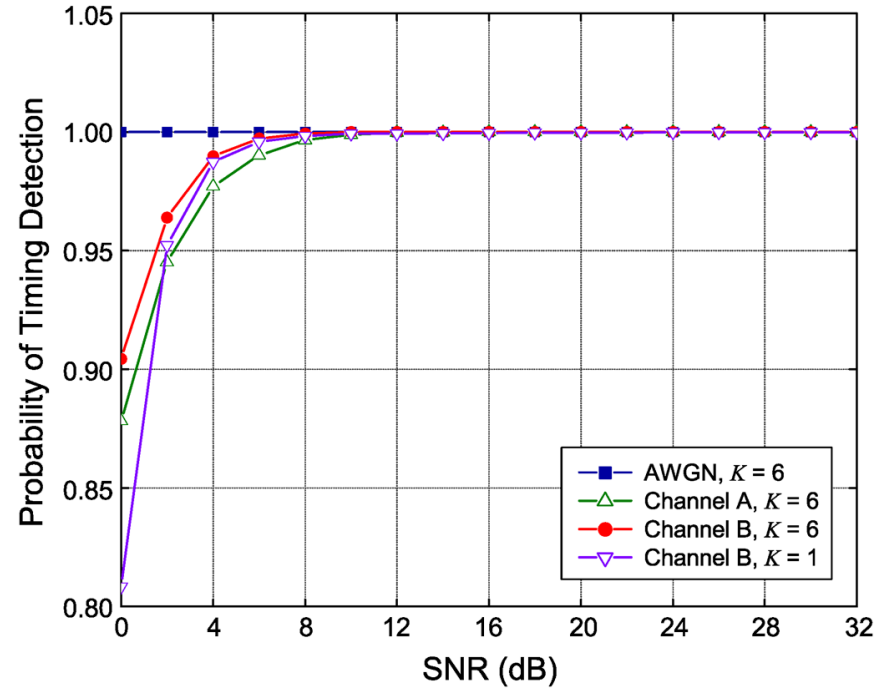

Fig. 5. Probability of detection for timing synchronization in the cooperation phase.

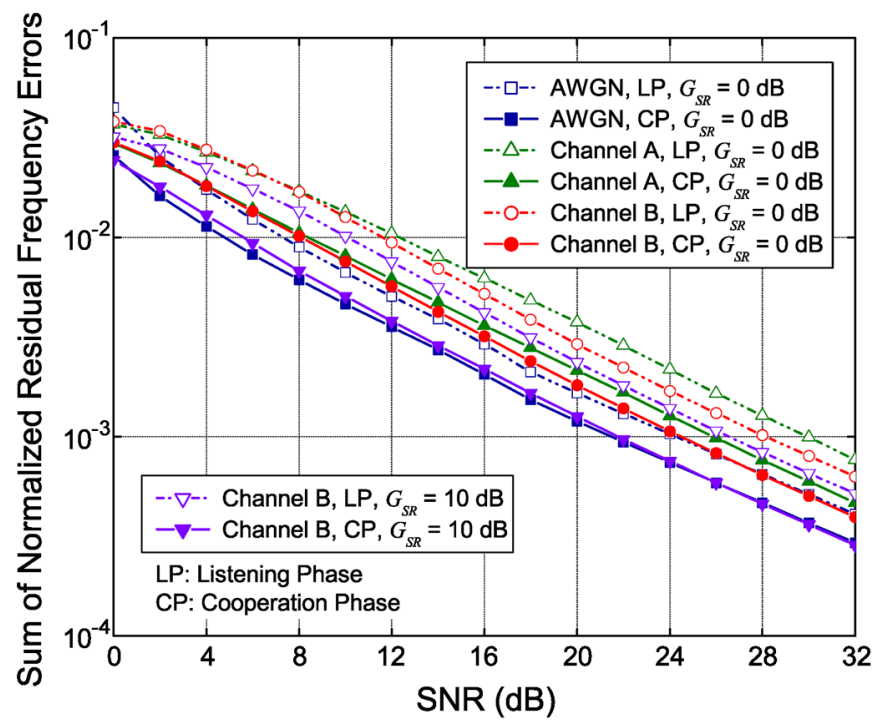

Fig. 6. Sum of normalized residual frequency errors after frequency synchronization.

Fig. 7 shows the normalized mean square error (NMSE) of the initial channel estimates in the cooperation phase, when perfect synchronization is assumed, and $G_{R D}=0 \mathrm{~dB}$. The NMSE in the cooperation phase is defined as

$$
\mathrm{NMSE} \triangleq \sum_{k \in \mathbb{\Pi}_{u}}\left|\frac{H_{S D}[k]-\hat{H}_{S D}[k]}{H_{S D}[k]}\right|^{2}+\left|\frac{H_{R D}[k]-\hat{H}_{R D}[k]}{H_{R D}[k]}\right|^{2} .
$$




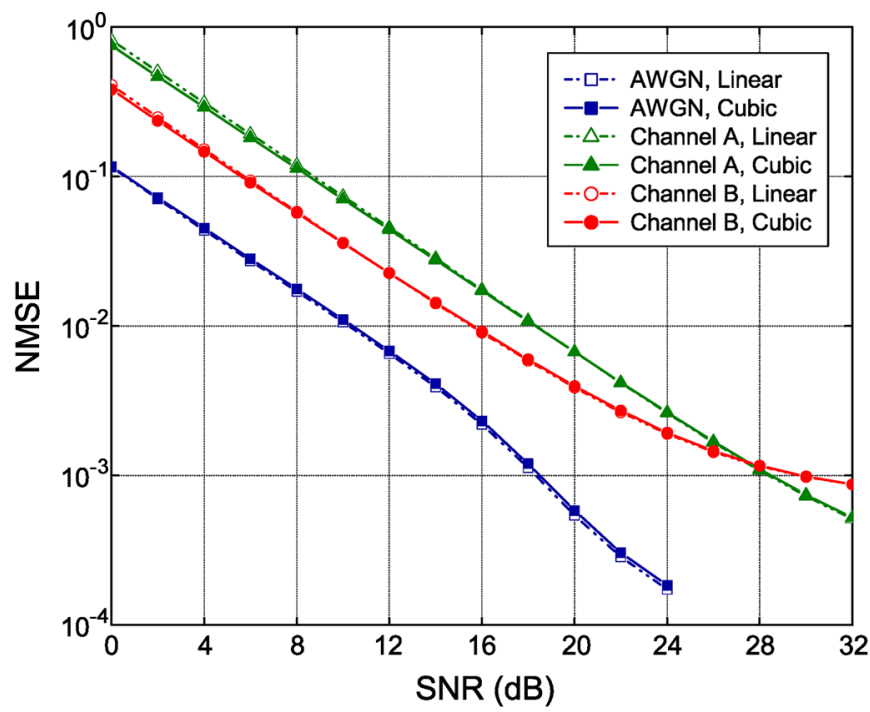

Fig. 7. NMSE of initial channel estimates in the cooperation phase.

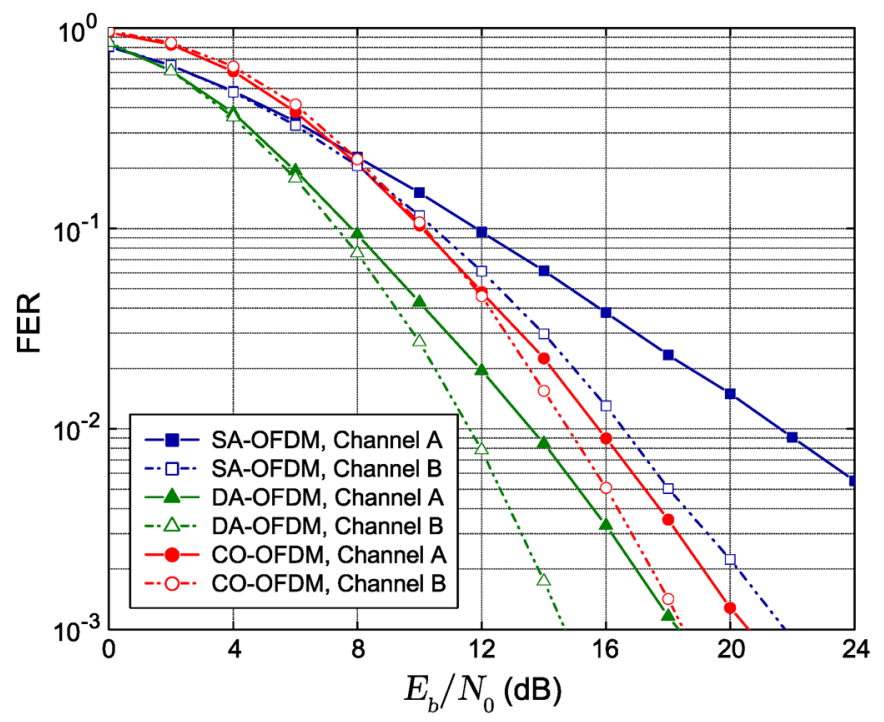

Fig. 8. FER performance under the assumptions of perfect synchronization and channel estimation.

For all channels, the log of the NMSE is found to decrease almost linearly with the SNR. The linear and cubic interpolation functions for (32) are shown to provide indistinguishable performance.

In Fig. 8, the overall frame-error-rate (FER) performance of the CO-OFDM system is compared with that of SA-OFDM and DA-OFDM systems under idealistic assumptions of perfect synchronization with zero timing offset and zero frequency offsets, and perfect channel estimation. The geometric gains are assumed to be $G_{S R}=10 \mathrm{~dB}$ and $G_{R D}=0 \mathrm{~dB}$. As expected, the CO-OFDM system is shown to provide significant performance improvement over the SA-OFDM system, particularly at high $E_{b} / N_{0}$. In particular, from the slopes of the FER curves, we notice that the CO-OFDM system achieves a diversity order comparable to that of the DA-OFDM system. The discrepancy between the CO-OFDM and DA-OFDM systems is due to energy consumption in the listening phase and higher rate error correction coding for the CO-OFDM system. The smaller

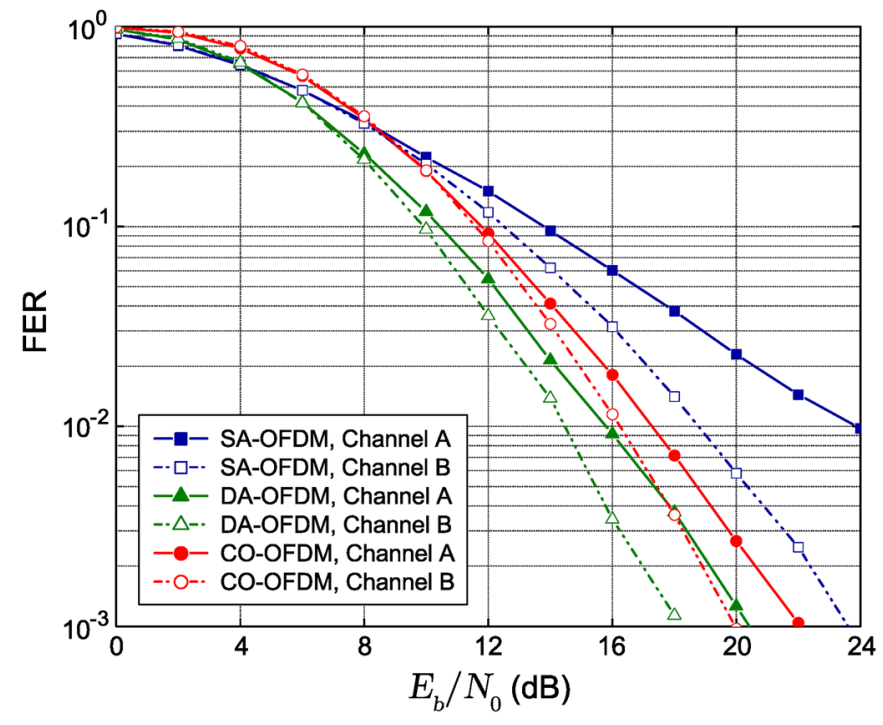

Fig. 9. FER performance with the proposed synchronization and channel estimation algorithms.

performance improvement from SA-OFDM to CO-OFDM in channel B compared to in channel A is because channel B has the higher frequency diversity to start with. This suggests that spatial diversity due to cooperation is more effective for an adverse environment with severe fading.

Fig. 9 shows the FER performance, when the proposed synchronization and channel estimation algorithms are adopted. The geometric gains are assumed to be the same as in Fig. 8 . It is assumed that the timing offset $\zeta$ is an integer value uniformly distributed over $[-3,3]$ samples and that the initial frequency error of each node is uniformly distributed over $[-2.0,2.0] \mathrm{ppm}$, as in Figs. 5 and 6. Linear interpolation is used for the initial channel estimation in the cooperation phase. We can still observe significant performance improvement of the CO-OFDM system over the SA-OFDM system. At a target FER of $10^{-2}$, for example, the energy gain of the CO-OFDM system over the SA-OFDM system is found to be as much as $6.7 \mathrm{~dB}$ for channel $\mathrm{A}$ and $2.5 \mathrm{~dB}$ for channel B. Fig. 10 shows the separate effect of synchronization and channel estimation on the overall performance of the CO-OFDM system in channel A. The figure shows that the impact of synchronization error is more significant than that of initial channel estimation error. This is partly because the residual frequency offset affects the phase tracking of channel estimates, even though the initial channel estimates are perfect.

The effects of geometric gains on the FER performance of the CO-OFDM system in channel A are illustrated in Fig. 11. The other simulation conditions are the same as Fig. 9. Note that the larger geometric gain $G_{S R}$ leads to the higher probability of successful decoding at the relay in the listening phase, resulting in a higher chance of cooperation. Numerical results in Fig. 11 show that $G_{S R}=10 \mathrm{~dB}$ is large enough to achieve the most of the cooperative diversity gain with negligible loss compared to the case of larger $G_{S R}$. On the other hand, the geometric gain $G_{R D}$ has a direct influence on the total received signal power in the cooperation phase. This explains why the performance improves continuously with $G_{R D}$ in Fig. 11 . 


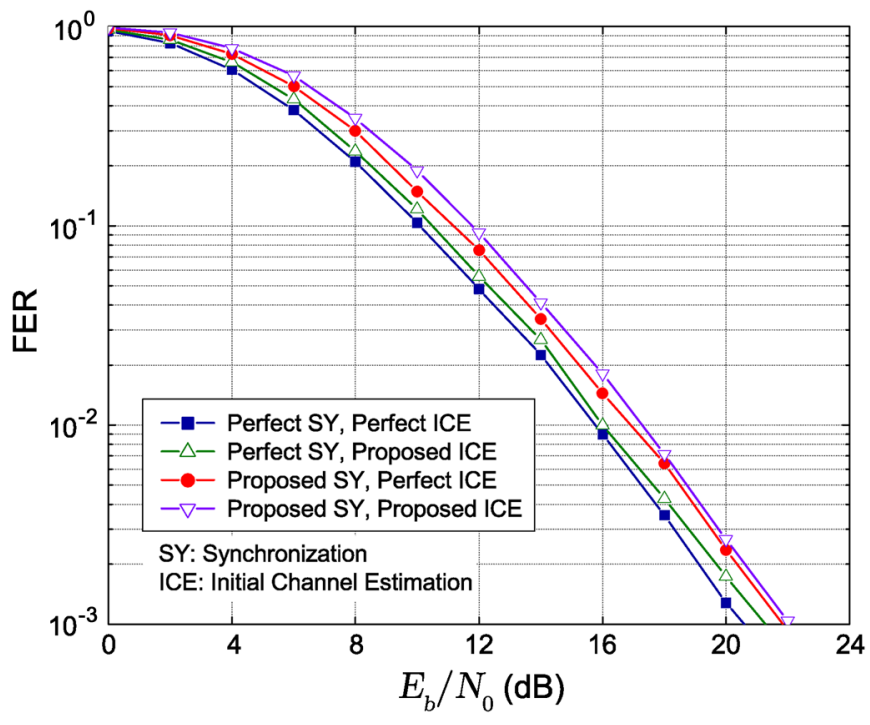

Fig. 10. Impacts of synchronization and channel estimation on the FER of the CO-OFDM system in channel A.

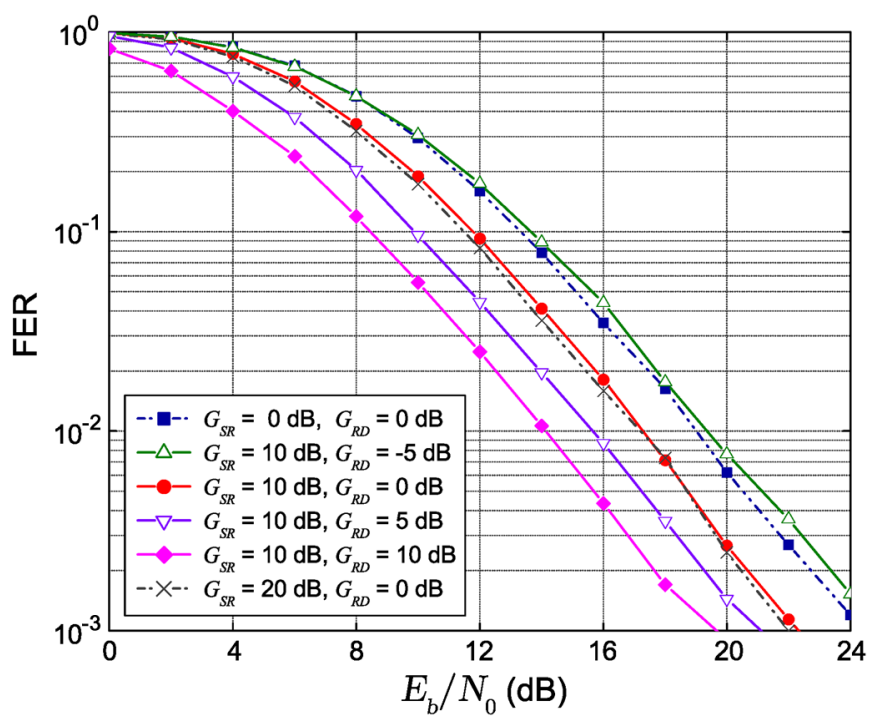

Fig. 11. Impacts of geometric gains on the FER of the CO-OFDM system in channel A.

\section{CONCLUSION}

We have designed a space-time cooperative system based on OFDM, referred to as a CO-OFDM system, and evaluated the system performance. Our design includes a two-phase cooperation protocol, as well as a transmitter and receiver architecture. We have also developed a frame structure, on which we have built synchronization and channel estimation algorithms suitable for the CO-OFDM system. Simulation results have demonstrated that the CO-OFDM system achieves a diversity gain comparable to the conventional space-time-coded OFDM system, even when synchronization and channel estimation are taken into account. For a channel with little frequency diversity, when the proposed synchronization and channel estimation algorithms are employed, the CO-OFDM system has shown to provide as much as $6.7 \mathrm{~dB}$ energy gain over an SA-OFDM system at a target FER of $10^{-2}$. We have also discussed the impacts of geometric gains on the FER performance.

\section{REFERENCES}

[1] V. Tarokh, N. Seshadri, and A. R. Calderbank, "Space-time codes for high data rate wireless communication: Performance criterion and code construction," IEEE Trans. Inf. Theory, vol. 44, no. 2, pp. 744-765, Mar. 1998.

[2] S. M. Alamouti, "A simple transmit diversity technique for wireless communications," IEEE J. Sel. Areas Commun., vol. 16, no. 8, pp. 1451-1458, Oct. 1998.

[3] A. Sendonaris, E. Erkip, and B. Aazhang, "User cooperation diversityPart I: System description," IEEE Trans. Commun., vol. 51, no. 11, pp. 1927-1938, Nov. 2003.

[4] A. Sendonaris, E. Erkip, and B. Aazhang, "User cooperation diversityPart II: Implementation aspects and performance analysis," IEEE Trans. Commun., vol. 51, no. 11, pp. 1939-1948, Nov. 2003.

[5] A. Nosratinia, T. E. Hunter, and A. Hedayat, "Cooperative communication in wireless networks," IEEE Commun. Mag., vol. 42, no. 10, pp. 74-80, Oct. 2004.

[6] J. N. Laneman and G. W. Wornell, "Distributed space-time coded protocols for exploiting cooperative diversity in wireless networks," IEEE Trans. Inf. Theory, vol. 49, no. 10, pp. 2415-2425, Oct. 2003.

[7] J. N. Laneman, D. N. C. Tse, and G. W. Wornell, "Cooperative diversity in wireless networks: Efficient protocols and outage behavior," IEEE Trans. Inf. Theory, vol. 50, no. 12, pp. 3062-3080, Dec. 2004.

[8] P. Mitran, H. Ochiai, and V. Tarokh, "Space-time diversity enhancements using space-time collaboration," IEEE Trans. Inf. Theory, vol. 51, no. 6 , pp. 2041-2057, Jun. 2005.

[9] H. Ochiai, P. Mitran, and V. Tarokh, "Design and analysis of collaborative diversity protocols for wireless sensor networks," in Proc. IEEE Veh. Technol. Conf.-Fall, Los Angeles, CA, Sep. 2004, pp. 4645-4649.

[10] T. M. Cover and A. A. El Gamal, "Capacity theorems for the relay channel," IEEE Trans. Inf. Theory, vol. IT-25, no. 5, pp. 572-584, Sep. 1979.

[11] R. U. Nabar, H. Bölcskei, and F. W. Kneubühner, "Fading relay channels: Performance limits and space-time signal design," IEEE J. Sel. Areas Commun., vol. 22, no. 6, pp. 1099-1109, Aug. 2004.

[12] D. L. Goeckel and Y. Hao, "Space-time coding for distributed antenna arrays," in Proc. IEEE Int. Conf. Commun., Paris, France, Jun. 2004, pp. 747-751.

[13] X. Li, "Space-time coded multi-transmission among distributed transmitters without perfect synchronization," IEEE Signal Process. Lett., vol. 11, no. 12, pp. 948-951, Dec. 2004.

[14] R. V. Nee and R. Prasad, OFDM for Wireless Multimedia Communications. London, U. K.: Artech House, 2000.

[15] IEEE, Part 11: Wireless LAN Medium Access Control (MAC) and Physical Layer (PHY) Specifications: High-Speed Physical Layer in the $5 \mathrm{GHz}$ Band, Sep. 1999, IEEE Std 802.11a-1999.

[16] I. Koffman and V. Roman, "Broadband wireless access solutions based on OFDM access in IEEE 802.16," IEEE Commun. Mag., vol. 40, no. 4, pp. 96-103, Apr. 2002.

[17] P. H. Moose, "A technique for orthogonal frequency division multiplexing frequency offset correction," IEEE Trans. Commun., vol. 42, no. 10, pp. 2908-2914, Oct. 1994.

[18] J. van de Beek, M. Sandell, and P. O. Börjesson, "ML estimation of time and frequency offset in OFDM systems," IEEE Trans. Signal Process., vol. 45, no. 7, pp. 1800-1805, Jul. 1997.

[19] T. M. Schmidl and D. C. Cox, "Robust frequency and timing synchronization for OFDM," IEEE Trans. Commun., vol. 45, no. 12, pp. 1613-1621, Dec. 1997.

[20] J. G. Proakis, Digital Communications, 4th ed. New York: McGrawHill, 2001.

[21] O.-S. Shin, H. T. Kung, and V. Tarokh, "Construction of block orthogonal Golay sequences and application to channel estimation of MIMO-OFDM systems," IEEE Trans. Commun., May 2007, to be published.

[22] D. Bertsekas and R. Gallager, Data Networks, 2nd ed. Englewood Cliffs, NJ: Prentice-Hall, 1992.

[23] O.-S. Shin, V. Tarokh, and H. T. Kung, "Space-time collaborative software radio OFDM physical layer design," Harvard Univ., Cambridge, MA, Tech. Rep. 05-001, Jan. 2005.

[24] R. L. Peterson, R. E. Ziemer, and D. Borth, Introduction to Spread Spectrum Communications. Englewood Cliffs, NJ: Prentice-Hall, 1995.

[25] G. V. Rangaraj, M. R. Raghavendra, and K. Giridhar, "Improved channel estimation for OFDM based WLAN systems," in Proc. Int. Conf. Wireless Commun. Netw., Chennai, India, Jun. 2003, pp. C27-C31.

[26] European Telecommunications Standards Institute (ETSI), Universal Mobile Telecommunications System (UMTS): Selection Procedures for the Choice of Radio Transmission Technologies for the UMTS, Apr. 1998. V3.2.0. 


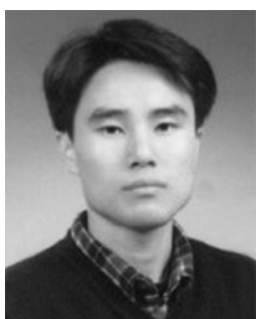

Oh-Soon Shin received the B.S., M.S., and Ph.D. degrees in electrical engineering from Seoul National University, Seoul, Korea, in 1998, 2000, and 2004, respectively.

From 2004 to 2005, he was with the Division of Engineering and Applied Sciences, Harvard University, Cambridge, MA, as a Postdoctoral Fellow. Since April 2006, he has been a Senior Engineer at Samsung Electronics, Suwon, Korea. His research interests include communication theory, wireless communication systems, and signal processing for communications.

Dr. Shin received the Best Paper Award from the CDMA International Conference in 2000.

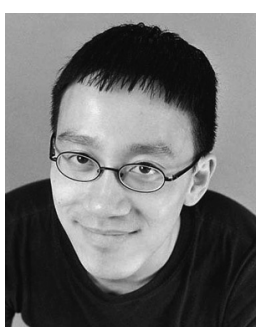

Albert M. Chan (S'96-M'06) received the B.A.Sc. degree from the University of Toronto, Toronto, ON, Canada, in 1997 and the S.M. and Ph.D. degrees from the Massachusetts Institute of Technology (MIT), Cambridge, in 1999 and 2004, respectively, all in electrical engineering.

During his studies, he spent leaves at Motorola, Mansfield, MA, in 1998, Ericsson, Inc., Research Triangle Park, NC, in 2000, and Agere Systems, Murray Hill, NJ, in 2001. In 2004, he was a Postdoctoral Fellow with the Signal, Information, and Algorithms Group at MIT. Since 2004, he has been a member of Technical Staff at Vanu, Inc., Cambridge. His research interests include signal processing and wireless communication systems. He authored a number of papers in these areas and has several patents pending.

Dr. Chan received the MIT Frederick C. Hennie III Award for Teaching Excellence, a Natural Sciences and Engineering Research Council of Canada Postgraduate Scholarship, the General Electric Canada Special Award in Recognition of Engineering Leadership, and a Canada Scholarship.

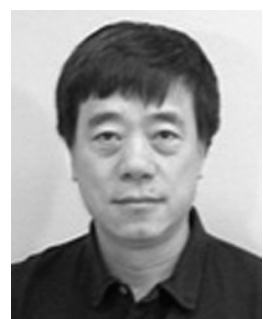

H. T. Kung received the $\mathrm{Ph} . \mathrm{D}$. degree from Carnegie Mellon University, Pittsburgh, PA, in 1974 and the B.S. degree from National Tsing Hua University, Hsinchu, Taiwan, R.O.C., in 1968.

$\mathrm{He}$ is currently William H. Gates Professor of Computer Sciences and Electrical Engineering, Harvard University, Cambridge, MA. Since 1999, he has co-chaired a joint Ph.D. program with the Harvard Business School on Information, Technology, and Management. He served on the faculty of Carnegie Mellon University from 1974 to 1992. He has pursued a variety of interests over his career, including complexity theory, database theory, systolic arrays, very-large-scale-integration design, parallel computing, computer networks, network security, and wireless communications.

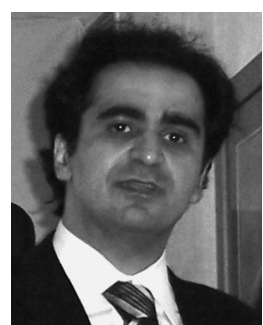

Vahid Tarokh (M'95-SM'03) received the Ph.D. degree in electrical engineering from the University of Waterloo, Waterloo, ON, Canada, in 1995.

$\mathrm{He}$ is currently a Perkins Professor of applied mathematics and Hammond Vinton Hayes Senior Fellow of electrical engineering at Harvard University, Cambridge, MA, where he defines and supervises research in communications, networking, and signal processing.

Dr. Tarokh has been one of the "Top 10 Most Cited Authors in Computer Science" since 2003, according to the ISI Web of Science. He is the recipient of a number of major awards and two honorary degrees. 